\title{
Explaining variance of avian malaria infection in the wild: the importance of host density, habitat, individual life-history and oxidative stress
}

\author{
Caroline Isaksson ${ }^{1,2^{*}}$, Irem Sepil ${ }^{1}$, Vladimer Baramidze ${ }^{1}$ and Ben C Sheldon ${ }^{1}$
}

\begin{abstract}
Background: Avian malaria (Plasmodium sp.) is globally widespread, but considerable variation exists in infection (presence/absence) patterns at small spatial scales. This variation can be driven by variation in ecology, demography, and phenotypic characters, in particular those that influence the host's resistance. Generation of reactive oxygen species (ROS) is one of the host's initial immune responses to combat parasitic invasion. However, long-term ROS exposure can harm the host and the redox response therefore needs to be adjusted according to infection stage and host phenotype. Here we use experimental and correlational approaches to assess the relative importance of host density, habitat composition, individual level variation and redox physiology for Plasmodium infection in a wild population of great tits, Parus major.

Results: We found that $36 \%$ of the great tit population was infected with Plasmodium (22\% P. relictum and 15\% P. circumflexum prevalence) and that patterns of infection were Plasmodium species-specific. First, the infection of P. circumflexum was significantly higher in areas with experimental increased host density, whereas variation in P. relictum infection was mainly attributed to age, sex and reproduction. Second, great tit antioxidant responses total and oxidizied glutathione - showed age- , sex- and Plasmodium species-specific patterns between infected and uninfected individuals, but reactive oxygen metabolites (ROM) showed only a weak explanatory power for patterns of $P$. relictum infection. Instead ROM significantly increased with Plasmodium parasitaemia.
\end{abstract}

Conclusions: These results identify some key factors that influence Plasmodium infection in wild birds, and provide a potential explanation for the underlying physiological basis of recently documented negative effects of chronic avian malaria on survival and reproductive success.

Keywords: Antioxidants, Glutathione, Host density, Hydroperoxides, Oxidative stress, Parus major, Plasmodium

\section{Background}

Avian malaria (Plasmodium $s p$ ) is a globally widespread disease but considerable variation exists in both prevalence and parasitaemia at small spatial scales [1,2]. This variation can be generated by several ecological, demographical and immunological mechanisms, including variation in vector and host densities and host susceptibility. For example, temperature, altitude and proximity to water bodies are small-scale ecological factors known to influence vector (e.g., mosquitoes [Culex, Aedes, and Culiseta], and black

\footnotetext{
* Correspondence: Caroline.Isaksson@biol.lu.se
'Department of Zoology, Edward Grey Institute, University of Oxford, Oxford,

'Department of Zoology, Edward Grey Institute, University of Oxford, Oxford, United Kingdom

${ }^{2}$ Current address: Evolutionary ecology group, Department of Biology, Lund University, Lund, Sweden
}

flies [Simuliidae]) and/or parasite distribution and abundance [2-8]. This in turn increases the probability of transmission from vector to host. Furthermore, if an area with malaria transmission is of poor quality (e.g., has low availability and/or quality of food), this may reduce the bird's condition and ability to resist a parasitic infection [9-11], further compounding the rate of infection. Similarly, although transmission rate of vector borne diseases are predicted to be independent of host density [12], high densities of birds may reduce the availability of food or other resources, thereby further impairing resistance. Other demographic parameters that may influence infection rates include sex and age structures of the population arising from differences in behaviour, reproductive effort and immune capacities [13,14]. 
The impact of avian malaria on wild birds has been difficult to estimate, especially in areas where avian malaria is common and the host-parasites have co-evolved. However, recent long-term and experimental studies have shown that there are effects on both survival and reproductive success [15-18]. On naive hosts such as island species and captive birds in zoo the impacts have been devastating [19]. For example on the Hawaiian Islands there was an accidental introduction of Plasmodium relictum and one of its vectors which caused direct die-offs during the acute infection state which continues to play a significant role in species distribution and is a serious threat for endangered species [19-22].

In the early stages of infection, the first line of defense is the host's innate, non-specific immune defence. Immune cells generate and release reactive oxygen species (ROS, commonly referred to as oxidative burst) that attack the parasite $[23,24]$. The advantage of oxidative burst is that it can act within minutes after transmission, in contrast to the acquired immune defence, which needs longer to develop the target-specific antibodies. However, the non-specificity and general cytotoxicity of ROS make it impossible for the host to distinguish between self and parasite tissue, causing oxidative damage to all types of adjacent molecules, which if not repaired, can lead to haemolysis and cellular dysfunction (i.e., a surplus of oxidants to antioxidants is commonly referred to as oxidative stress). If the parasite resists the host's ROS attack and starts to multiply and grow, it also starts to generate ROS as a by-product of haemoglobin digestion [25]. Consequently, the detrimental and beneficial effects of ROS need to be fine-tuned by the host's antioxidant defences during different stages of infection [26,27], which can result in a quadratic relationship between oxidative stress and malaria severity. This association can depend on other host- and environment-specific characters, including age and nutrition which influences the oxidative status [28-30].

Here we assess the relative importance of ecological (habitat quality and spring date), demographic (host density, age and sex), life-history (reproduction and body mass) and physiological (total and oxidized glutathione which are the most important intra-cellular antioxidant system and reactive oxygen metabolites [ROM]) factors for natural variation in Plasmodium infection and parasitaemia (i.e. parasite density) in a population of great tits, Parus major. The sampling took place in the spring, which suggests that most infections are relapse infections from previous autumn, since most vectors have not yet emerged [31]. Assessment of the relative importance of a broad range of factors, from ecology to physiology, to explain variation in patterns of avian malaria infection in the wild has rarely been done [11], but is important for understanding the significance of effects found under controlled laboratory situations. In addition, the present population allows test of experimentally increased host density on patterns of prevalence.

\section{Results}

In the present population of great tits, 107 of the 299 screened birds were infected with Plasmodium (36\%); 65 with $P$. relictum (prevalence of $22 \%$ ) and 44 with P. ciricumflexum (prevalence of 15\%) morphospecies; only two birds had mixed infection (see Table 1 for sample sizes). The infection for first year breeders was $17 \%$ for both morphospecies, and the corresponding figures for older birds were $26 \%$ and $14 \%$, for, P.relictum and $P$. ciricumflexum, respectively (see below for analysis of age-dependence).

\section{Infection of Plasmodium relictum}

In Table 2, the top ten models are presented along with the averaged model estimates for the parameters included (all models with $\Delta<4$ ). For $P$. relictum infection, there were only small differences in the AICc between the top ten models (Table 2), so no model could be confidently singled out as the best. However, five out of the ten parameters were important for explaining variation in $P$. relictum infection; namely age, sex, clutch size, spring date and ROM. The experimental manipulation of density was not important in any of the top ten models for P. relictum (Figure 1). The raw data from the top three parameters revealed a $12 \%$ increase in infection from first year to older breeders and males were less infected (17\%) than females (26\%). In terms of clutch size, uninfected birds had laid a larger clutch size (mean \pm standard deviation: $9.23 \pm 1.49)$ compared to the infected birds (8.88 \pm 1.55). Regarding spring date, it should be mentioned that it was highly correlated to GSH:GSSG ratio ( $r=-0.293$, $\mathrm{p}<0.0001$, see Additional file 1) i.e., the earlier in the spring the more reduced to oxidized glutathione.

When interactions were included the top model retained $\mathrm{tGSH}$, age $\times \mathrm{tGSH}$ and sex $\times \mathrm{tGSH}$ apart from the parameters in Model 1 (sex, age and clutch size; see S2 for model details, Figure 2). Although not significant, the interactions suggest that young and male great tits have higher tGSH when infected compared to when uninfected; and vice versa for older and female birds.

Table 1 Sample sizes of Plasmodium infected and uninfected individuals

\begin{tabular}{lccccc}
\hline & \multicolumn{2}{c}{ P. circumflexum } & & \multicolumn{2}{c}{ P. relictum } \\
\cline { 2 - 3 } \cline { 6 - 6 } & Uninfected & Infected & & Uninfected & Infected \\
\hline Young & $\mathrm{n}=106$ & $\mathrm{n}=21$ & & $\mathrm{n}=105$ & $\mathrm{n}=22$ \\
Older & $\mathrm{n}=144$ & $\mathrm{n}=23$ & & $\mathrm{n}=124$ & $\mathrm{n}=43$ \\
Female & $\mathrm{n}=140$ & $\mathrm{n}=23$ & & $\mathrm{n}=121$ & $\mathrm{n}=42$ \\
Male & $\mathrm{n}=112$ & $\mathrm{n}=21$ & & $\mathrm{n}=110$ & $\mathrm{n}=23$ \\
\hline
\end{tabular}


Table 2 Summary of AIC modelling of Plasmodium relictum infection in great tits

\begin{tabular}{|c|c|c|c|c|c|c|}
\hline & Parameters* & Deviance & AICc & $\triangle \mathrm{AICC}$ & $\omega$ & \\
\hline Model 1 & $1+2+9$ & 267.23 & 277.47 & 0 & 0.01 & \\
\hline Model 2 & $1+2+4+8+9$ & 263.20 & 277.66 & 0.19 & 0.01 & \\
\hline Model 3 & $1+2+8+9$ & 265.40 & 277.74 & 0.27 & 0.01 & \\
\hline Model 4 & $1+2+4+9$ & 265.40 & 277.74 & 0.27 & 0.01 & \\
\hline Model 5 & $1+2+4+8$ & 265.61 & 277.95 & 0.48 & 0.01 & \\
\hline Model 6 & $2+4+8$ & 268.04 & 278.28 & 0.81 & 0.01 & \\
\hline Model 7 & $2+4+8+9$ & 266.06 & 278.4 & 0.93 & 0.01 & \\
\hline Model 8 & $1+2+8$ & 268.17 & 278.41 & 0.94 & 0.01 & \\
\hline Model 9 & $1+2+4$ & 268.25 & 278.49 & 1.02 & 0.01 & \\
\hline Model 10 & $1+2+5+9$ & 266.17 & 278.51 & 1.04 & 0.01 & \\
\hline Null model & Intercept & 276.01 & 280.06 & 2.59 & 0.00 & \\
\hline \multicolumn{7}{|c|}{$\begin{array}{l}\text { * see code below for which parameters } \\
\text { that are included }\end{array}$} \\
\hline \multicolumn{7}{|c|}{ Averaged model parameters: } \\
\hline Parameters & Code & Coeff. & SE & Low Cl & Upp Cl & $\begin{array}{l}\text { Rel. } \\
\text { import. }\end{array}$ \\
\hline (Intercept) & & 2.49 & 3.14 & -3.66 & 8.65 & \\
\hline Age(young) & 1 & -0.40 & 0.39 & -1.16 & 0.35 & 0.69 \\
\hline Cs & 2 & -0.13 & 0.13 & -0.37 & 0.12 & 0.68 \\
\hline $\mathrm{D}(\mathrm{low})$ & 3 & 0.00 & 0.12 & -0.23 & 0.23 & 0.12 \\
\hline Spring date & 4 & -0.04 & 0.05 & -0.13 & 0.06 & 0.52 \\
\hline GSSG & 5 & 0.04 & 0.11 & -0.17 & 0.25 & 0.25 \\
\hline Mass & 6 & -0.04 & 0.12 & -0.28 & 0.19 & 0.26 \\
\hline $\mathrm{Q}($ low) & 7 & 0.00 & 0.15 & -0.29 & 0.29 & 0.13 \\
\hline ROM & 8 & -0.13 & 0.18 & -0.49 & 0.23 & 0.54 \\
\hline Sex(male) & 9 & -0.28 & 0.35 & -0.97 & 0.40 & 0.56 \\
\hline $\mathrm{tGSH}$ & 10 & 0.04 & 0.10 & -0.17 & 0.24 & 0.26 \\
\hline
\end{tabular}

The top ten models are presented along with relative parameter importance (based on a model average with $\triangle \mathrm{AICC}>4$ ).

cs clutch size, $D$ breeding density, GSSG oxidized glutathione, $Q$ habitat quality, $R O M$ reactive oxygen metabolites, $t G S H$ total glutathione, Rel. imp relative parameters importance, $\mathrm{Cl}$ confidence interval (lower and upper), $\omega$ model weight.

\section{Infection of Plasmodium circumflexum}

In Table 3, the top ten models are presented along with the averaged model estimates for the parameters included. For $P$. circumflexum, the manipulation of bird density was the best explanatory variable, followed by GSSG (Table 3). In line with our prediction, great tits breeding in high density areas showed a twofold increase in prevalence (18\%) compared to birds breeding in the low density areas (9\%). Moreover, P. circumflexum infected birds had a lower GSSG than uninfected birds (mean std GSSG \pm standard deviation, uninfected: $0.01 \pm 0.96$, infected: $-0.27 \pm 0.91$, Figure 3$)$. When interactions were added, the best fitting model included density, GSSG, age and agexdensity (see S3 for model details, Figure 1). Closer examination revealed that the density pattern was

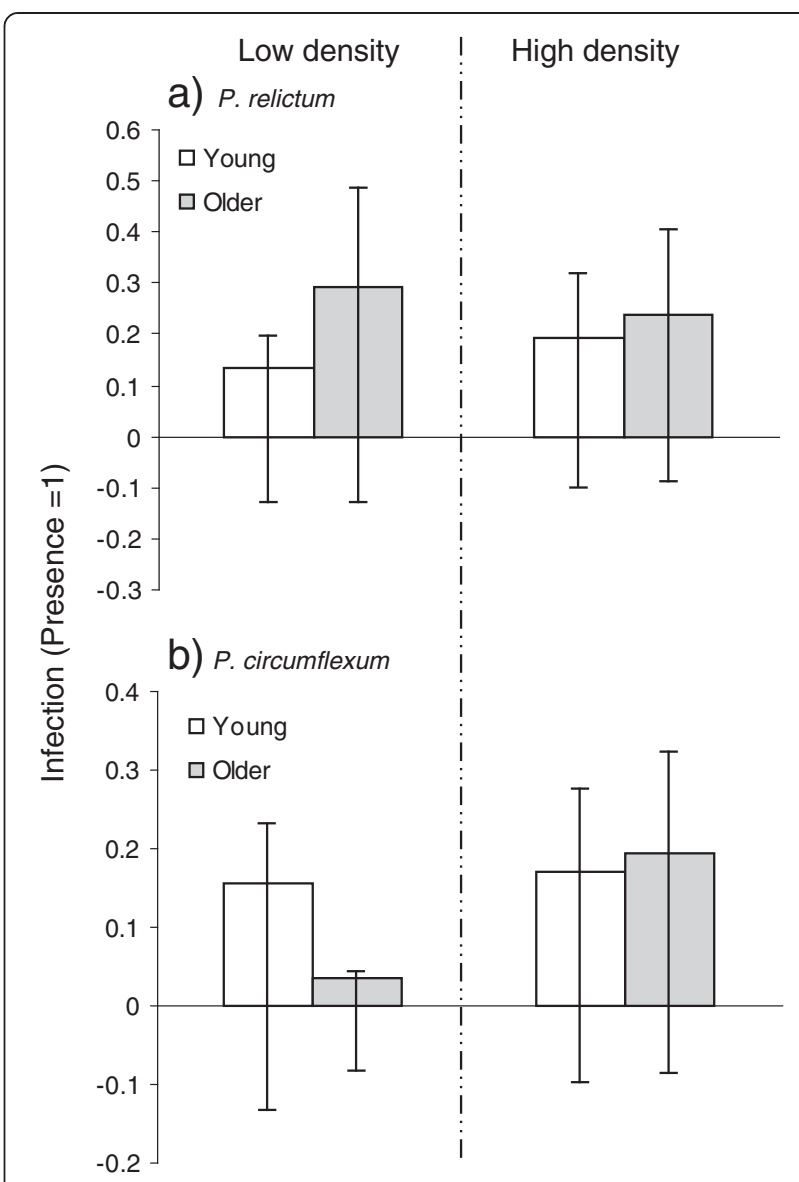

Figure 1 Plasmodium infection in relation to breeding density and age. Infection of $\boldsymbol{a})$ P. relictum, and $\boldsymbol{b}$ ) P. circumflexum in adult great tits (first year breeders and older) in relation to experimental manipulation of breeding density $(\mathrm{LD}=$ low density, $\mathrm{HD}=$ high density). Mean \pm Confidence Intervals $(\mathrm{Cl})$, presence $=1$ and absence $=0$, i.e., the higher value the larger proportion is infected.

found to be stronger in older great tits, with $3 \% P$. circumflexum infection in low density compared to $19 \%$ in the high density habitats. For first year breeders the $P$. circumflexum infection was $18 \%$ in both densities.

In addition, parasite density of both Plasmodium species was quantified. However, the species-specific models did not explain any significant variation in parasitaemia, so will not be presented. There were no significant associations between species-specific parasitaemia and any of the physiological variables $(\mathrm{F}=0.14-2.48, \mathrm{p}=0.71-0.11)$. Although we are aware of the difference in virulence of the two different Plasmodium species [23], the digestion of haemoglobin by both species releases iron $\left(\mathrm{Fe}^{2+}\right.$ and $\mathrm{Fe}^{3+}$ ) which can generate ROM [24-26] (see also Methods). A correlational analysis were performed and it revealed the predicted positive association between ROM and parasitaemia $(\mathrm{n}=93, \mathrm{r}=0.25, \mathrm{p}=0.018)$, but not for tGSH $(\mathrm{n}=100, \mathrm{r}=-0.00, \mathrm{p}=0.990)$, GSSG 


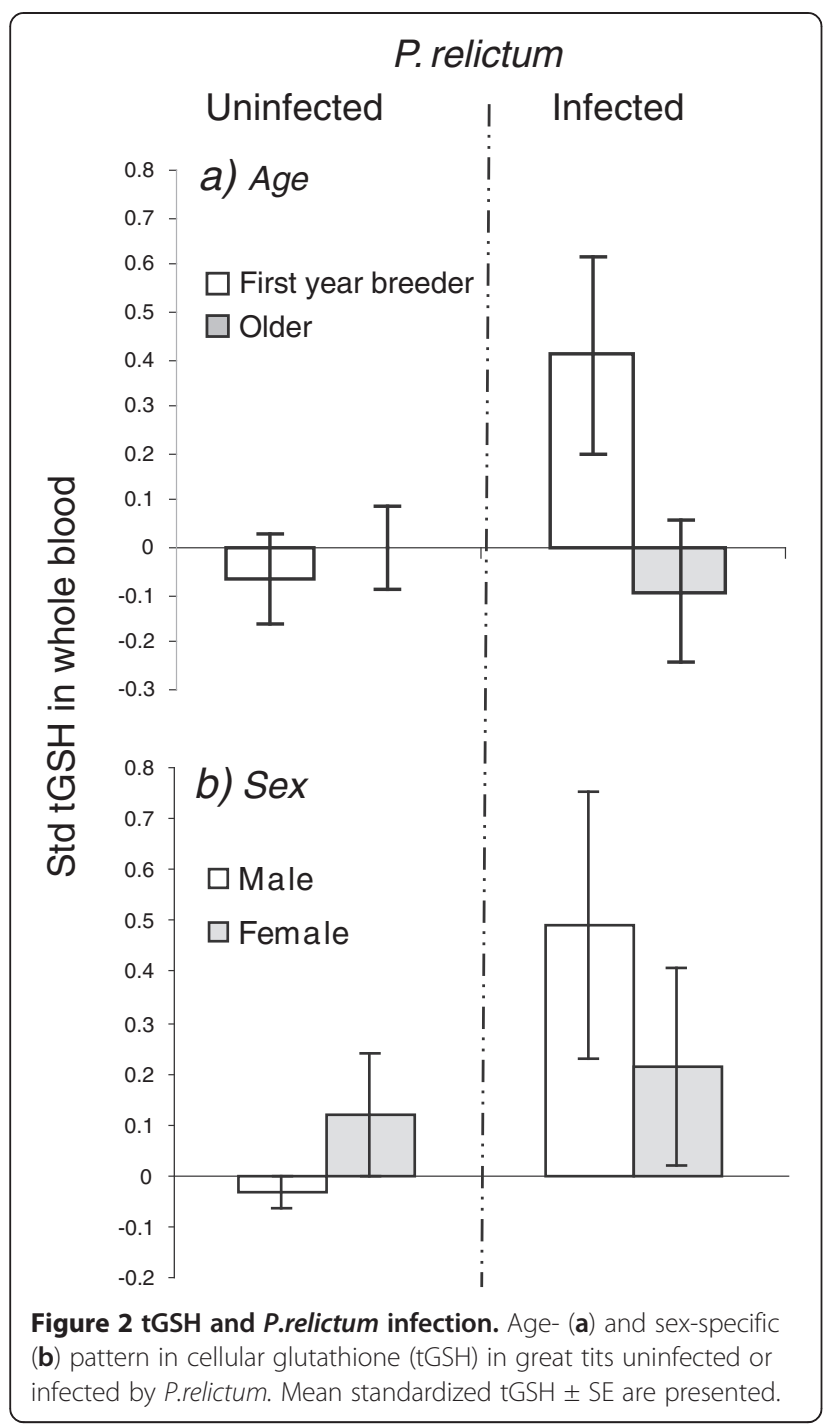

$(\mathrm{n}=100, \mathrm{r}=-0.05, \mathrm{p}=0.605)$ and the GSSG:tGSH ratio $(\mathrm{n}=100, \mathrm{r}=-0.14, \mathrm{p}=0.178)$.

\section{Discussion}

In the present study, we combined experimental manipulation of host density across two contrasting environments with assessment of host physiology and life-history in a natural population of great tits to understand causes of variation in Plasmodium infection. The key and novel findings of this study were, firstly, that manipulation of host density increases $P$. circumflexum infection, but not infection with $P$. relictum; and secondly; that host antioxidants and ROM physiology show species-specific patterns in relation to infection, and thirdly, that ROM increases with Plasmodium parasitaemia.

\section{Avian malaria, ecology and demography}

Host density was found to be the only ecological and demographic parameter of importance for $P$. circumflexum
Table 3 Summary of AIC modelling of Plasmodium circumflexum infection in great tits

\begin{tabular}{|c|c|c|c|c|c|c|}
\hline & Parameters* & Deviance & $\mathrm{AICC}$ & $\Delta \mathrm{AlCc}$ & $\omega$ & \\
\hline Model 1 & $3+5$ & 207.98 & 216.14 & 0.00 & 0.07 & \\
\hline Model 2 & 3 & 210.49 & 216.58 & 0.44 & 0.05 & \\
\hline Model 3 & $1+3+5$ & 207.27 & 217.52 & 1.38 & 0.03 & \\
\hline Model 4 & $3+5+8$ & 207.47 & 217.72 & 1.58 & 0.03 & \\
\hline Model 5 & $3+5+10$ & 207.57 & 217.82 & 1.68 & 0.03 & \\
\hline Model 6 & $1+3$ & 209.71 & 217.88 & 1.74 & 0.03 & \\
\hline Model 7 & $3+4+5$ & 207.73 & 217.98 & 1.84 & 0.03 & \\
\hline Model 8 & $2+3+5$ & 207.76 & 218.01 & 1.87 & 0.03 & \\
\hline Model 9 & $3+8$ & 209.98 & 218.14 & 2.00 & 0.02 & \\
\hline Model 10 & $3+5+9$ & 207.90 & 218.14 & 2.01 & 0.02 & \\
\hline Null model & Intercept & 226.63 & 230.67 & 4.34 & 0.01 & \\
\hline \multicolumn{7}{|c|}{$\begin{array}{l}\text { * see code below for which parameters } \\
\text { that are included }\end{array}$} \\
\hline \multicolumn{7}{|c|}{ Averaged model parameters: } \\
\hline Parameters & Code & Coeff. & SE & $\begin{array}{c}\text { Low } \\
\mathrm{Cl}\end{array}$ & $\begin{array}{l}\text { Upp } \\
\text { Cl }\end{array}$ & $\begin{array}{l}\text { Rel. } \\
\text { import. }\end{array}$ \\
\hline (Intercept) & & -1.48 & 1.76 & -4.93 & 1.97 & \\
\hline age(young) & 1 & 0.07 & 0.21 & -0.35 & 0.49 & 0.23 \\
\hline Cs & 2 & -0.01 & 0.05 & -0.11 & 0.09 & 0.16 \\
\hline $\mathrm{D}(\mathrm{low})$ & 3 & -1.03 & 0.44 & -1.90 & -0.16 & 1.00 \\
\hline $\begin{array}{l}\text { Spring } \\
\text { date }\end{array}$ & 4 & 0.00 & 0.02 & -0.04 & 0.05 & 0.17 \\
\hline GSSG & 5 & -0.18 & 0.22 & -0.61 & 0.24 & 0.59 \\
\hline Mass & 6 & -0.01 & 0.08 & -0.16 & 0.15 & 0.14 \\
\hline $\mathrm{Q}($ low $)$ & 7 & 0.01 & 0.16 & -0.30 & 0.32 & 0.13 \\
\hline ROM & 8 & -0.02 & 0.08 & -0.18 & 0.14 & 0.21 \\
\hline sex(male) & 9 & 0.01 & 0.13 & -0.25 & 0.28 & 0.13 \\
\hline tGSH & 10 & -0.02 & 0.09 & -0.19 & 0.15 & 0.18 \\
\hline
\end{tabular}

The top ten models are presented along with relative parameter importance (based on a model average with $\triangle \mathrm{AICC}>4$ ).

cs clutch size, $D$ breeding density, GSSG oxidized glutathione, $Q$ habitat quality , $R O M$ reactive oxygen metabolites, $t G S H$ total glutathione, $R e l$. imp relative parameters importance, $\mathrm{Cl}$ confidence interval (lower and upper), $\omega$ model weight.

infection. This link was absent for infection with P.relictum. This suggests that population density reduces the bird's ability to resist infection by $P$. circumflexum, the more virulent of the two Plasmodium species [32]. Possibly, this is via more intense competition over resources or it could be mediated via higher levels of testosterone or stress hormones in high density areas which have been shown to supress the immune system [33]. Alternatively, the vectors transmitting $P$. circumflexum either aggregate where hosts are abundant or that vectors carrying the parasite are able to infect several birds, whereas the vector transmitting $P$. relictum may be more widely distributed or only feed on one host at one point in time [33-35] but see [36].

In Wytham Woods; a woodland in the proximity of Bagley, recent work on great tits and the closely related 


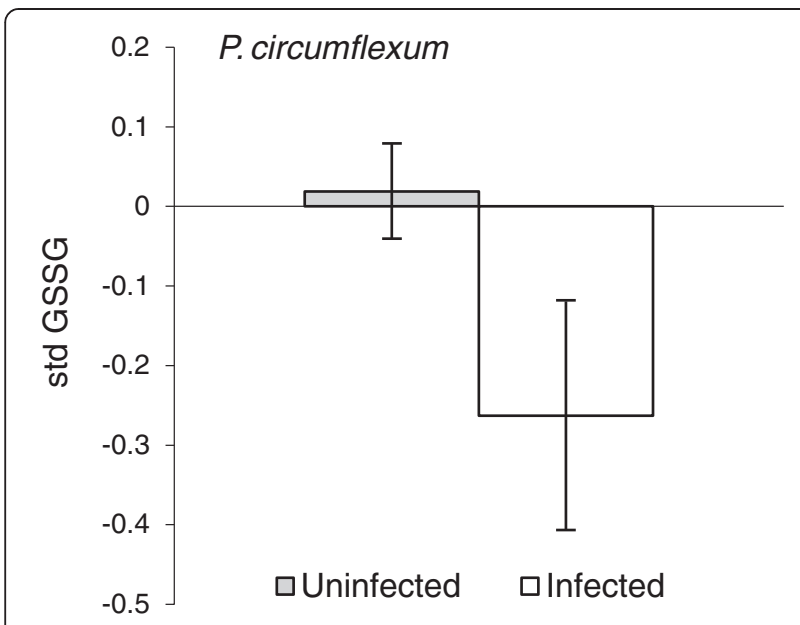

Figure 3 GSSG and $P$. circumflexum infection. Oxidized

glutathione (GSSG) in P. circumflexum infected and uninfected great tits. Mean standardized GSSG \pm SE are presented.

sympatric host species, blue tits (Cyanistes caeruleus), have shown that the two Plasmodium species have pronounced differences in their spatial distributions and impacts on both hosts species $[7,32]$. While $P$. circumflexum infections exhibit pronounced spatial structuring that is stable over years in both host species; $P$. relictum infections are effectively randomly distributed in space (Lachish et al. in press). Likewise, P. circumflexum infections are linked with reduced survival, particularly during the acute stage of infection [32]; whereas $P$. relictum infections are associated with reproductive costs [7]. Hence the species-specific relationships between ecological factors and parasite prevalence detected in the present study are not unexpected. Moreover, in Wytham woods it was shown that territory size was linked to host density i.e., the larger territory the lower density. This estimate of host density did not explain patterns of Plasmodium infection in the conspecific blue tits, Cyanistes caeruleus [1]. Similar to the present study, however, a positive association was found between large-scale breeding density (i.e., measured as number of nests within a $1500 \mathrm{~m}$ radius) and Leucocytozoons prevalence and parasitaemia in nestling eagle owls (Bubo bubo) [37]. In temperate regions Plasmodium species show a bimodal peak in infection, one in autumn and one in spring, and the spring peak (measured here) is most often a relapse from the autumn infection gained in the previous year $[31,38]$. Since juvenile great tits disperse during late summer-early autumn, the impact of the current environment on first-year breeders may be small compared to the older great tits which have strong site fidelity. Indeed, the association of host density manipulation was stronger among the older great tits for infection with $P$. circumflexum. In contrast to our prediction, habitat quality and spring date did not explain much (or any) variation in infection patterns in either of the two morphospecies.

\section{Avian malaria, life-history and oxidative stress}

There was no overall important life-history or oxidative stress parameter that explained variation in infection of both morphospecies. For $P$. relictum infection, age was one of the best predictors and in accordance to our prediction; older great tits were to a greater extent infected compared to younger great tits. However, there was no sign of an age effect on $P$. circumflexum infection, which is possibly due to the strong influence of host density (see above). Naturally, the probability of getting bitten by an infected vector increases with time regardless of species [1]. Despite that, older individuals may have an acquired immune defence that is familiar to Plasmodium parasites, so an overall decline in cellular mechanisms and resistance with age can be an explanation for the influence of age [39]. Indeed, since most birds are likely to have a relapse infection, the low tGSH in old $P$. relictum infected birds can be a result of a more rapid decline of the antioxidant defences (or indirectly via degradation of other cellular mechanisms affecting GSH synthesis) compared to uninfected ageing birds. For first year breeders, tGSH was higher when infected compared to those that were not infected, which is what we predicted during a chronic malaria infection. Alternatively, young and old individuals have different hormetic responses to parasites [40] or selective disappearance of the old individuals with a high tGSH in response to infection. The cause for this age difference in $\mathrm{tGSH}$ in response to infection warrants further investigation.

Given the difference between the sexes in behaviour and physiology during the breeding season, it was not surprising that sex explained some variation in P.relictum infection [41]. Generally, in both mammals and birds, males are to a greater extent infected by malaria, but in the present population females have slightly higher presence of infection, but not significantly. Similarly, in a nearby forest, Wytham woods, female blue tits showed a significantly higher presence of infection of P. relictum [7]. Possibly, the direction the association is a result of the timing of sampling i.e., breeding season (discussed below). Furthermore, there was an interaction between sex and tGSH, revealing that infected males have a higher tGSH compared to uninfected males, whereas infected females have lower (or no difference) tGSH compared to uninfected females. Interestingly, sex shows a similar pattern to age with regards to the interaction (see above, and Figure 2). The group with relatively lower presence of infection (males and young) had higher tGSH when infected compared to the uninfected birds for the same group, whereas the group with relatively higher presence of infection (females and older) had lower tGSH when 
infected. This sex- and age-specific response of tGSH to $P$. relictum parasites may play a role in susceptibility to this infection.

Alternatively, and independently of the similar age pattern, the investment into reproduction by infected females is associated with a greater physiological costs compared to uninfected females and compared to males, thus, females may not be able to up-regulate their antioxidant system [11]. Recently, a study of great tits revealed that increasing brood size results in an increase in malaria parasitaemia in males, but that both sexes show a decrease in resistance to oxidative stress i.e., red blood cell resistance to an external free radical attack [42]. However, a direct link between oxidative stress and parasitaemia was not found. Here clutch size was an important predictor of $P$. relictum infection, with uninfected birds having larger clutch size than infected birds, supporting the previously found fitness cost [32]. ROM was slightly higher in the uninfected birds, thus the production of hydroperoxyl radicals when exposed to oxidizing agent is less likely in $P$. relictum prevalent birds. This seem to be in contrast to overall parasitaemia, where ROM increases with parasite density $(\mathrm{p}=0.018)$, when analysed separately per species only a trend was found ( $P$. relitum: $\mathrm{p}=0.12, P$. circumflexum: $\mathrm{p}=0.11$ ). As mentioned above, the overall effect of parasitaemia may be independent of parasite species virulence but rather a result of parasite metabolism [43]. When a parasite digests haemoglobin it releases haem with iron in its ferrous state $\left(\mathrm{Fe}^{2+}\right)$. This state is highly reactive and can easily oxidize to $\mathrm{Fe}^{3+}$ and thereby generate ROM [43-45]. Thus, increased parasite metabolism and abundance may be followed by increased oxidative damage (due to increased generation of hydroperoxyl radicals) unless the antioxidant defence is adequate. In a recent study of Seychelles warbler (Acrocephalus sechellensis), malaria infection was linked to a higher ROM, but parasitaemia was not measured [11]. An alternative explanation for the patterns in both Seychelles warblers and great tits is that high generation of hydroperoxyl radicals may be linked to other unmeasured abiotic or biotic factors that increase the susceptibility to generate them, resulting in a positive association between avian malaria and ROM.

In contrast to $P$. relictum, GSSG (oxidized GSH) rather than the tGSH explained variation in $P$. circumflexum prevalence. Generally, a high GSSG indicates that the cell experiences an oxidative challenge, and that the GSH antioxidant system is in action [46]. However, in an infected cell the antioxidant efficiency of GSH has been shown to be suppressed by a down-regulation of glutathione peroxidase and a low GSSG can be detected even though the oxidative challenge is high [47], see also [48]. This is perhaps the most likely interpretation of the lower GSSG in P. circumflexum prevalent birds. However, it should be noted that in the present study all erythrocytes were measured i.e. not only the infected ones. Thus, the redox environment for the parasite may be different to the average host cell GSH/GSSG redox homeostasis. Possibly, the individuals with low GSSG are more susceptible to $P$. cirumflexum infection or there has been selective disappearance of individuals with high intracellular GSSG independent of infection. In captivity, healthy partridges GSSG show a quadratic relationship with age [49]. Unfortunately, the present study is not able to disentangle the effect of age and infection on GSSG and GSH. The species difference can be a result of different manipulations of the host GSH system, parasite metabolism or virulence $[14,50,51]$.

\section{Conclusion}

The present study is the first experimental test showing that bird breeding density increases infection of an avian malaria species - $P$. circumflexum. Patterns of $P$. relictum infection, on the other hand, were better explained by host factors: sex, age and clutch size. As in human malaria, GSH redox system seems to form a part of the physiological response to avian malaria, but in a species-specific pattern and depending on the bird's age and sex. However, independent of Plasmodium species and host factors, Plasmodium abundance showed a positive association with generation of ROM. This could form part of a mechanistic explanation for the recently documented negative impact of infection on fitness in passerines [15,16,32] and suggest that oxidative stress may be an important cost of infection in wild populations. Future experimental studies will be important for determining the causal relationship between the different avian malaria species and parameters of oxidative stress.

\section{Methods}

\section{Ethical consideration}

All work was carried out within UK standard requirements (Home office license number PL30/2659) and the guidelines of the University of Oxford. All methods were approved by the University of Oxford ethical review board and permission to work in Bagley Woods was obtained from the property owner's - St. John's College/ Oxford. Birds were caught by nest box traps and then kept in individual cloth bags and sampled within half an hour. All blood samples were taken by C.I (Personal license PIL 30/8503).

\section{Study site}

This study was carried out during April-June in 2009 in a nest-box population of great tits (Parus major). The study site, Bagley woods, is a 250 -ha woodland $\left(51^{\circ} 42^{\prime} \mathrm{N}\right.$, $5^{\circ} 37^{\prime} \mathrm{W}$ ), consisting of a mixed matrix of plantations of either deciduous trees (mainly oak Quercus dominated, 
high quality habitat $=\mathrm{HQ}, \mathrm{n}_{\text {plots }}=8$ ) or evergreen trees (mainly pine Pinus and larch Larix, low quality = LQ, $\mathrm{n}_{\text {plots }}=4$ ) (see map in Additional file 2). High quality and low quality classification is based on abundance of caterpillars in deciduous areas, crucial food source for great tits during spring time [52]. In early 2007, nest boxes ( $\mathrm{n}=513$ ) were experimentally placed at two densities (high density $=\mathrm{HD}, 68$ nest boxes, $\mathrm{n}_{\text {plots }}=6$, and low density $=\mathrm{LD}, 18$ nest boxes, $\mathrm{n}_{\text {plots }}=6$ ) in similar sized areas (between 9-12 ha). These arrangements produce average densities of 23.3 great tit nests per HD area and 12.3 great tit nests per LD area (i.e., an approximate two-fold increase in density). Birds nest according to the box availability, which creates plots of different bird densities. Although some natural nests are likely to occur within the study site, these are unlikely to affect the treatment densities to any great extent (only one natural nest was found, CI personal observation). In this way, a two-factor design is created, with replicated manipulations. There were no difference between the high and low breeding densities in clutch size $(\mathrm{F}=0.89$, $\mathrm{p}=0.344)$, condition $(\mathrm{F}=2.33, \mathrm{p}=0.128)$ and spring date $(\mathrm{F}=1.06, \mathrm{p}=0.306)$. Breeding close to standing water bodies can be an important determinant for avian malaria infection due to higher abundance of vectors [1]. The only water basins within the present wood arise from sporadic truck tracks after heavy rain; these are, as far as we are aware, distributed at random throughout the wood.

\section{Study species and sampling}

Adult great tits were captured with nest box traps while feeding their chicks (between day 6-10 after hatching). In total 299 birds were captured $\left(\mathrm{n}_{\text {females }}=164, \mathrm{n}_{\text {males }}=135\right.$, see Tab. 1). Among the older birds, 131 of 168 (78\%) were known (based on capture history) to have bred in the study site the previous year (2008), and only 10 (7.6\%) individuals had moved to a different density plot between 2008 and 2009. Regarding the first year breeders, 56 out of 126 (44\%) had been ringed as nestlings in Bagley in the previous year. For five birds age was not determined.

Approximately $110 \mu$ l blood was drawn from the jugular vein with a heparinised syringe. For malaria screening, a few drops of blood were stored in SET buffer (0.015 M $\mathrm{NaCl}, 0.05 \mathrm{M}$ Tris, 0.001 M EDTA, pH 8.0) and saved in $-20^{\circ} \mathrm{C}$. Then total genomic DNA was extracted using standard ammonium acetate method and stored in $\mathrm{AE}$ Buffer (Qiagen) until disease identification (see below). For the oxidative stress assays, two different approaches were used: for GSH assays, $40 \mu$ l blood was diluted with $2 \mu \mathrm{l}$ EDTA and then immediately frozen in liquid nitrogen in the field. The rest (approximately $60 \mu \mathrm{l}$ ) was kept cool on ice until it was centrifuged two to three hours later, and then frozen in liquid nitrogen. For proper separation between plasma and red blood cells samples were centrifuged at $1800 \mathrm{rpm}$ for 10 minutes. At the end of each field day all samples were transferred to a $-80^{\circ} \mathrm{C}$ freezer. All the biochemical assays were run within 6 months after collection and within the same week to reduce variation in degradation among the samples [53].

\section{Malaria screening}

A quantitative polymerase chain reaction (qPCR)-based assay was used to amplify and quantify a portion of the Plasmodium cytochrome b gene [15]. Total DNA concentration was measured using a Picogreen assay (Quant-iT Picogreen dsDNA Assay Kit, Invitrogen) and molecular conversion calculations, based on the size and base composition of the DNA fragment, were used to estimate DNA copy number in this solution see [40]. The samples were diluted to $2 \mathrm{ng} / \mu \mathrm{l}$. qPCR amplifications were performed in $25 \mu \mathrm{l}$ reactions, with the following final concentrations: 12.5 $\mu$ of Super Mix (Platinum SYBR Green qPCR SuperMix-UDG, Invitrogen), 10 ng of diluted DNA and $0.2 \mu \mathrm{M}$ of primers L9 (5'-AAACAATTCCTAACAAAA CAGC-3') and New R (5'-ACATCCAATCCATAATAAAG CA-3'). The primers were carefully designed to ensure Plasmodium-specific amplification and to enable the differentiation of the two Plasmodium morphospecies that are common in the study site, $P$. relictum and $P$. circumflexum for more details see $[7,32]$. The temperature profile included a pre-incubation step at $50^{\circ} \mathrm{C}$ for $2 \mathrm{~min}$ and an initial denaturation step at $95^{\circ} \mathrm{C}$ for $2 \mathrm{~min}$, followed by 43 cycles of denaturation at $95^{\circ} \mathrm{C}$ for $15 \mathrm{sec}$, annealing at $56^{\circ} \mathrm{C}$ for $30 \mathrm{sec}$ and extension at $72^{\circ} \mathrm{C}$ for $30 \mathrm{sec}$. The reactions were run on an Mx3000P machine (Stratagene) and SYBR Green detection system was employed. Each sample was run in triplicate and Plasmodium copy number was estimated by calculating the mean value for the triplicate.

\section{Oxidative stress assays}

\section{Total and oxidized glutathione (tGSH and GSSG)}

Glutathione (GSH) is the most abundant intra-cellular antioxidant, and probably the most important. It targets hydrogen peroxide $\left(\mathrm{H}_{2} \mathrm{O}_{2}\right)$ which is the major oxidant produced during oxidative burst and parasite metabolism [54,55]. The oxidized form of GSH - glutathione disulphide (GSSG) can be reduced back to its active form (GSH) by a nicotinamide adenine dinucleotide phosphate (NADPH) dependent reaction and glutathione reductase (GR), and it is this redox-cycle that the quantification method of tGSH and GSSG is based on [56]. With minor modifications, tGSH and GSSG assays were conducted according to Baker et al. [56] and Vandeputte et al. [57], adapted for birds in Isaksson et al. [58]. Briefly, $4 \mu \mathrm{L}$ of whole blood was diluted with $16 \mu \mathrm{L} 5 \%$ 5-sulfoasalicylic acid (SSA). To precipitate proteins, all samples were then centrifuged at $8000 \mathrm{~g}$ for $10 \mathrm{~min}$ at $4^{\circ} \mathrm{C} .10 \mu \mathrm{L}$ of the sample was further diluted with $400 \mu \mathrm{L}$ GSH buffer 
(143 $\mathrm{mM} \mathrm{NaH} \mathrm{PO}_{4}$ and $6.3 \mathrm{mM}$ EDTA, pH 7.4). For GSSG assay, $200 \mu \mathrm{l}$ of the sample were treated immediately with $1 \mu \mathrm{l}$ of 4-vinylpyridine (4-vp) and incubated for 1 hour at $37^{\circ} \mathrm{C}$. The reaction mixture (prepared fresh daily) contain GSH buffer, 5.5'-dithio-bis (2-nitrobenzoic acid) (DTNB) and NADPH. A 96-well plate was prepared for both tGSH (diluted plasma without 4-vp) and GSSG (diluted sample incubated with 4-vp) by adding, $20 \mu \mathrm{l}$ sample and $200 \mu \mathrm{l}$ reaction mix, and then $2 \mu \mathrm{l}$ of $0.34 \mathrm{U} / \mathrm{ml}$ and $0.17 \mathrm{U} / \mathrm{ml}$ GR added for tGSH and GSSG, respectively. All samples were run in duplicates and the colour generated by 2-nitro-5-thiobenzoic acid (the product from the reaction between DTNB and GSH) was immediately measured on a SPECTRA MAX 190 plate reader at $412 \mathrm{~nm}$ during 5 minutes. The obtained changes (kinetic mode) were compared with daily made GSH and GSSG standards of known concentrations. All chemicals used were purchased from Sigma-Aldrich, UK or Melford, UK. The assays were further validated by measuring a subset of individuals twice. The across and within-assay repeatability was tested by using a restricted maximum likelihood (REML) mixed-model with individual identity as a random factor. The across-assay repeatability for $\mathrm{tGSH}$ was $78 \%$ (tGSH, $\mathrm{n}=54$, variance components \pm SE, ID: $0.29 \times 10^{-3} \pm 0.09 \times 10^{-3}$, residual: $\left.0.08 \times 10^{-3} \pm 0.02 \times 10^{-3}\right)$, and for GSSG it was $71 \%$ (GSSG, $\mathrm{n}=48$, variance components \pm SE, ID: $10.60 \times 10^{-6} \pm 3.79 \times 10^{-6}$, residual: $4.22 \times 10^{-6} \pm 1.23^{\times} 10^{-6}$ ). Within-assay repeatabilities were $98 \%$ and $95 \%$, respectively. Furthermore, GSH concentration is higher in red blood cells compared to extra-cellular plasmatic fluids (Adult great tits: Mean $(\mu \mathrm{M}) \pm \mathrm{SD}$; red blood cells: $15.36 \pm 5.86$, plasma: $3.56 \pm 1.81)$. Thus, to control for the number of red blood cells in the blood, haemoglobin concentration was measured using Drabkin's regent (0.05 g KCN, $0.2 \mathrm{~g} \mathrm{~K}_{3} \mathrm{Fe}(\mathrm{CN})_{6}$ and $1 \mathrm{~g} \mathrm{NaHCO}_{3}$ for 1 liter $\mathrm{dH}_{2} \mathrm{O}$ ). However, neither haematocrit nor haemoglobin showed a significant effect on tGSH (hematocrit; $\mathrm{n}=303$, $\mathrm{F}=0.048, \mathrm{p}=0.826$, haemoglobin; $\mathrm{n}=284, \mathrm{~F}=0.019$, $\mathrm{p}=0.890)$, thus was not considered any further. Laboratory day significantly influenced both tGSH and GSSG ( $\mathrm{p}<0.001$ ), thus measures needed to be standardized (see data handling and statistics).

\section{Reactive Oxygen Metabolites (ROM)}

ROMs are generated by oxidation of hydroperoxides $(\mathrm{ROOH})$. This oxidation occurs in the early phase of an oxidative cascade, which means that ROMs can further cause damage by generating more toxic ROS [55]. Therefore ROMs can directly related to the amount of ROS and susceptibility to ROS [55,59]. The d-ROMs test (Diacron, Grosseto, Italy) was used to detect the generated ROM in plasma. With minor modifications, the protocol provided by the manufacturer was followed.
Briefly, a master mix of 1:100 of chromogen and acidic buffer was prepared, and $200 \mu \mathrm{l}$ were added to each well on a 96-microplate. $4 \mu \mathrm{l}$ of plasma was added and then incubated at $37^{\circ} \mathrm{C}$ for $75 \mathrm{~min}$. Samples were analysed at $490 \mathrm{~nm}$ (Biotech Power, Northwest Science LTD). Each plate contained blank, calibrator, and standard plasma sample. The final d-ROM calculations were expressed as $\mathrm{mg} \mathrm{H}_{2} \mathrm{O}_{2} / \mathrm{dl}$. For d-ROM assay the acrossassay repeatability was $93 \%(\mathrm{~d}-\mathrm{ROM}, \mathrm{n}=64$, variance components \pm SE: $I D=3.79 \pm 0.29$, residual $=0.28 \pm 0.05$ ), and within assay repeatability was $99 \%$. Recently, ROM was shown to be sensitive to time of sampling [60]. Here, however, time did not influence ROM concentration $\left(\mathrm{F}_{1,243}=0.89, \mathrm{p}=0.35\right)$, and was therefore not controlled for.

\section{Data handling and statistics}

\section{Parameters and the rational for inclusion in models}

The aim of the present study is to investigate which factors that explain variance in malaria infection in great tits and their relative importance (see below). The factors included are described in detail below. Two environmental factors were explored: habitat quality (evergreen versus deciduous-tree dominated plots) and spring date. Since the present study was conducted in the spring (before most vectors emerge), the majority of infections are likely to be relapses from previous infections [31]. However, later in the spring the likelihood of obtaining a primary infection increases. Thus, we expect Plasmodium infection to increase with spring date. For habitat quality, we predict that birds breeding in the low quality (ever-green) habitats to have a higher infection rate, since these birds are generally in poorer condition [52].

Five demographic and host intrinsic parameters were explored: host density - an increase in Plasmodium infection was predicted as an indirect effect of high host density [1,12,34]. Sex - previous results show mixed results. Thus, no overall directional sex effect can be predicted [1,42]. Age - over time the probability to be bitten by an infected vector is likely to increase [7]. In addition, parasite resistance declines with age due to immunosenescence [61]. Based on that we predicted older birds to have a higher proportion of infected individuals than the first year breeders [1]. Body mass - we predicted infected birds had a have lower mass (see also above); and clutch size - chronic malaria infection has been related to fitness costs [32], hence we predicted that prevalent birds had a smaller clutch size.

Three oxidative stress parameters were explored; $t G S H$ if the majority of infections are relapses rather than primary infections (see above), we predicted tGSH to be up-regulated in infected birds [23,26,27]. Since nothing is yet known about $P$. circumflexum and $P$. relictum and 
GSH the prediction is based on P. falciparum and GSH. During primary infection the tGSH has been shown to be down-regulated to increase cellular ROS, thereby increasing the cellular oxidative stress. However, to have a high oxidative stress under a prolonged time is likely to be more damaging than beneficial for the host due the damaging nature of ROS to anything in its surroundings, thus tGSH is predicted to be at normal levels again. Potentially, it is higher than normal to protect the cell from ROS generated from parasite metabolism [54]. In addition, $P$. falciparum is known to control the intracellular tGSH and GSSG:tGSH redox homeostasis, thus during chronic infection it is most likely the interest of both host and parasite to have a high tGSH. Hence the prediction that tGSH is up-regulated. GSSG - is the oxidized form of GSH, thus a high GSSG would indicate a high antioxidant activity of GSH but also high exposure to ROS, we predicted that a relapse infection is positively associated with GSH redox activity (there was a negative association between GSH (reduced form) and GSSG, $\mathrm{r}=-0.626, \mathrm{p}<0.0001$ ), higher GSSG in prevalent birds compared to if uninfected is predicted. We did not include the GSH:GSSG ratio in the models due to its high correlation with spring date, tGSH and GSSG, however, as can be seen in the Additional file 3 there is no association with prevalence patterns when analyzed separately. $R O M$ - if chronic malaria infection is physiologically challenging (i.e., no up-regulation of tGSH or antioxidant activity of GSH) we predicted ROM to be higher in prevalent birds $[59,60]$. In addition, some key biologically relevant interactions were tested such as age $x$ antioxidant response and sex $\times$ clutch size. These are summarized in additional material (Additional files 4 and 5). All oxidative stress parameters were standardized for laboratory day to control for daily variation in chemical reaction rates (i.e., all solutions were made fresh and from scratch each day). Since a model selection approach was used (see below) to analyse the data, it is not appropriate to control for this by adding laboratory day as a covariate i.e., that would generate models that control and others that do not control for this artefact. Instead all concentrations were standardized for laboratory day by subtracting the lab day mean from the raw value and dividing that by the standard deviation of that day; this dimensionless quantity (the z-score) is commonly used in statistical analysis [62]. All samples were assayed randomly, thus it is unlikely that the standardization will bias the results.

\section{Akaike information criteria (AIC) modeling}

A model selection approach (AIC) was used to find the model (or models) that explain most of the variation found in infection (Tables 2 and 3). This analysis compares all possible combination of models in a given data set and ranks them by their AIC value; the model with the lowest AIC value is the best fitting model [63,64]. To account for the number of parameters included, AICc is a stricter estimate of AIC and therefore used here [63]. To evaluate the relative importance of single parameters we performed a model average calculation based on all models with a $\Delta$ value $<4(\Delta$ AIC $=$ AICc specific model minus the AICc from the best fitted model [lowest AICc]; [65], but see also Additional file 3). The relative likelihood of each model was estimated by normalized $\Delta$ AICc weights $(\omega)$, an index of relative plausibility. It should be noted that the model selection approach is not based on direct hypothesis-testing (although, we have predictions about the directions of the effects, see above); thus parameters included in the best fitted model do not necessarily have a significant effect. For Plasmodium infection, species-specific models were run (Tables 2 and 3). For the top ten models including the biologically relevant interactions, the estimates and confidence intervals are provided in the additional material (Additional files 4 and 5). Nest box was fitted as random effect and the model was run with binomial distribution for infection with logit link function. Initially, habitat plot (12 areas) was also included as a random factor, but since it only explained between $0-1 \%$ of variation it was removed to simplify models. There were no differences in the final results whether body mass or body condition (residuals from the regression between tarsus and mass) was included in the models. For simplification the raw data of mass was used instead of condition. All models were sensitive to missing values, thus only individuals with all target traits were included in the AIC approximation. The reduced sample sizes are given in Tables 2 and 3. The AIC modeling was performed in R 2.12.2 [66] and the other statistical tests in JMP 8 (SAS Institute. Inc., Cary, US).

\section{Additional files}

Additional file 1: Correlational analysis between all the covariates that are used in the model for Plasmodium prevalence. In addition, GSH:GSSG ratio are shown here, but since it correlates with spring date and GSSG and tGSH it was not included in the models.

Additional file 2: Schematic picture of the experimental design of breeding density in Bagley Woods/Oxfordshire. Each dot indicates a nest box and each habitat plot is separated with lines.

Additional file 3: This is the results from single parameter analysis using generalized linear models (GLM) with binomial distribution and logit link function. All parameters, except GSH:GSSG ratio, were included in the AIC models presented in the paper (Tables 2 and 3).

Additional file 4: $P$. relictum prevalence.

Additional file 5: $P$. circumflexum prevalence.

Competing interests

The authors declare that they have no competing interests. 


\section{Authors' contributions}

$\mathrm{Cl}$ and BS conceived and designed the study, $\mathrm{Cl}$ collected the data, $\mathrm{Cl}$ and VB performed the laboratory work. Cl and IS analysed the data. CI, IS and BS wrote the manuscript. All authors read and approved the final manuscript.

\section{Acknowledgement}

We are grateful to St. John's College/Oxford for allowing us to work on their property. We are grateful to Simon Evans, Teddy Wilkin, and Lucy Garrett for assistance with field work, Alicia Davies for help with malaria quantification, Iris Finkemeier for help with GSH protocol optimization, Colin Garroway for statistical advice and R-codes and Tobias Uller for comments on the manuscript. We are also grateful for technical services and equipment use in: Lee Sweetlove's lab and Gail Preston's at the Department of Plant Sciences (Oxford), and Angus Buckling's lab at Zoology (Oxford). For financial support we thank Wenner-Gren foundations (fellowship to C. Isaksson), and Governmental award of R. Georgia (MSc studentship to V. Baramidze).

Received: 23 July 2012 Accepted: 26 March 2013

Published: 8 April 2013

\section{References}

1. Wood MJ, Cosgrove CL, Wilkin TA, Knowles SCL, Day KP, Sheldon BC Within-population variation in prevalence and lineage distribution of avian malaria in blue tits, Cyanistes caeruleus. Mol Ecol 2007, 16:3263-3273

2. Lachish S, Knowles SCL, Alves R, Wood MJ, Sheldon BC: Infection dynamics of endemic malaria in a wild bird population: parasite speciesdependent drivers of spatial and temporal variation in transmission rates. J Anim Ecol 2011, 80:1207-1216.

3. Bensch $\mathrm{S}$, Akesson A: Temporal and spatial variation of hematozoans in Scandinavian willow warblers. J Parasitol 2003, 89:388-391. doi:10.1645/0022-3395(2003)089[0388:TASVOH]2.0.CO;2.

4. Valkiūnas G: Avian malaria parasites and other haemosporidia. Boca Raton: CRC Press; 2005

5. Rogers DJ, Randolph SE: Climate change and vector-borne diseases. Advan Parasitol 2006, 62:345-381.

6. Garamszegi LZ: Climate change increases the risk of malaria in birds. Glob Change Biol 2011, 17:1751-1759.

7. Knowles SCL, Wood MJ, Alves R, Wilkin TA, Bensch S, Sheldon BC: Molecular epidemiology of malaria prevalence and parasitaemia in a wild bird population. Mol Ecol 2011, 20:1062-1076.

8. Sehgal RNM, Buermann W, Harrigan RJ, Bonneaud C, Loiseau C, Chasar A, Sepil I, Valkiūnas G, lezhova T, Saatchi S, Smith TB: Spatially explicit predictions of blood parasites in a widely distributed African rainforest bird. Proc R Soc B 2010, 278:1025-1033.

9. Ricklefs RE, Swanson BL, Fallon SM, Martinez-Abrain A, Scheuerlein A, Gray J, Latta SC: Community relationships of avian malaria parasites in southern Missouri. Ecol Monogra 2005, 75:543-559.

10. Duckworth RA, Mendonca MT, Hill GE: A condition dependent link between testosterone and disease resistance in the house finch. Proc R Soc B 2001, 268:2467-2472.

11. van de Crommenacker J, Richardson DS, Kolz AM, Hutchings K, Komdeur J: Parasitic infection and oxidative status are associated and vary with breeding activity in the Seychelles warbler. Proc R Soc B 2011. doi:10.1098/rspb.2011.1865. online.

12. Anderson RM, May RM: Population biology of infectious diseases: Part I. Nature 1979, 280:361-367.

13. Sheldon $B C$, Verhulst $\mathrm{S}$ : Ecological immunology: costly parasite defences and trade-offs in evolutionary ecology. Trends Ecol Evol 1996, 11:317-321.

14. Sorci G, Faivre B: Inflammation and oxidative stress in vertebrate hostparasite systems. Phil Trans R Soc B 2009, 364:71-83.

15. Knowles SCL, Palinauskas V, Sheldon BC: Chronic malaria infections increase family inequalities and reduce parental fitness: experimental evidence from wild bird population. J Evol Biol 2010, 23:557-569.

16. Knowles SCL, Nakagawa S, Sheldon BC: Elevated reproductive effort increases blood parasitaemia and decreases immune function in birds: a meta-regression approach. Funct Ecol 2009, 23:405-415.

17. Asghar M, Hasselquist D, Bensch S: Are chronic avian haemosporidian infections costly in wild birds? J Avian Biol 2011, 42:530-537.

18. Opplinger A, Christe P, Richner H: Clutch size and malaria resistance. Nature 1996, 391:565.
19. LaPointe DA, Atkinson CT, Samuel MD: Ecology and conservation biology of avian malaria. Ann N Y Acad Sci 2011, 1249:211-226.

20. van Riper C, van Riper SGIII, Goff ML, Laird M: The epizootiology and ecological significance of malaria in Hawaiian land birds. Ecol Monog 1986, 56:327-344.

21. Yorinks $N$, Atkinson $C T$ : Effects of malaria (Plasmodium relictum) on activity budgets of experimentally-infected juvenile Apapane (Himatione sanguinea). Auk 2000, 117:731-738.

22. Atkinson CT, Samuel CT: Avian malaria Plasmodium relictum in native Hawaiian forest birds: epizootiology and demographic impacts on apapane Himatione sanguinea. J Avian Biol 2010, 41:357-366.

23. Stocker R, Hunt NH, Buffinton GD, Weidemann MJ, Lewis-Hughes PH, Clark IA: Oxidative stress and protective mechanisms in erythocytes in relation to Plasmodium vinckei load. Proc Natl Acad Sci U S A 1985, 82:548-551.

24. Swindle EJ, Metcalfe DD: The role of reactive oxygen species and nitric oxide in mast cell-dependent inflammatory responses. Immunol Rev 2007, 217:185-205.

25. Goldberg DE: Hemoglobin degradation. Curr Top Microbiol 2005, 295:275-291.

26. Lüersen K, Walter RD, Müller S: Plasmodium falciparum-infected red blood cells depend on a functional glutathione de novo synthesis attributable to an enhanced loss of glutathione. Biochem J 2000, 346:545-552

27. Müller S: Redox and antioxidant systems of the malaria parasite Plasmodium falciparum. Mol Microbiol 2004, 53:1291-1305.

28. Bertrand S, Criscuolo F, Faivre B, Sorci G: Immune activation increases the susceptibility to oxidative tissue damage in zebra finches. Funct Ecol 2006, 20:1022-1027.

29. Monaghan P, Metcalfe NB, Torres R: Oxidative stress as a mediator of life history trade-offs: mechanisms, measurements and interpretation. Ecol Lett 2009, 12:75-92.

30. Isaksson C, Sheldon BC, Uller T: The challenge of integrating oxidative stress into life-history biology. Bioscience 2011, 61:194-202.

31. Cosgrove CL, Wood MJ, Day KP, Sheldon BC: Seasonal variation in Plasmodium prevalence in a population of blue tits Cyanistes caeruleus. J Anim Ecol 2008, 77:540-548.

32. Lachish S, Knowles SCL, Alves R, Wood MJ, Sheldon BC: Fitness effects of endemic malaria infections in a wild bird population: the importance of ecological structure. J Anim Ecol 2011, 80:1196-1206.

33. Tracy RC, Nussear KE, Esque TC, Dean-Bradley K, Tracy CR, DeFalco LA, Castle $K T$, Zimmerman LC, Espinoza RE, Barber AM: The importance of physiological ecology in conservation biology. Integr Comp Biol 2006, 46:1191-1205.

34. Smith DL, Dushoff J, McKenzie FE: The risk of a mosquito-borne infection in a heterogeneous environment. Pub Lib Sci Biol 2004, 2:e368.

35. Palinauskas V, Valkiunas G, Bolshakov CV, Bensch S: Plasmodium relictum (lineage P-SGS1): Effects on experimentally infected passerine birds. Exp Parasitol 2008, 120:372-380

36. Kimura M, Darbro JM, Harrington LC: Avian malaria parasites share congeneric mosquito vectors. J Parasitol 2010, 96:144-151

37. Ortego J, Cordero PJ: Factors associated with the geographic distribution of leucocytozoa parasitizing nestling eagle owls (Bubo bubo): a local spatial-scale analysis. Conserv Genet 2010, 11:1479-1487.

38. Beaudoin RL, Applegate JE, David DE, McLean RG: A model for the ecology of avian malaria. J Wild Dis 1971, 7:5-13.

39. Sol D, Jovani $R$, Torres J: Parasite mediated mortality and host immune response explain age-related differences in blood parasitism in birds. Oecologia 2003, 135:542-547.

40. Mattson MP: Hormesis defined. Ageing Res Rev 2008, 7:1-7.

41. McCurdy DG, Shutler D, Mullie A, Forbes MR: Sex-biased parasitism of avian hosts: relations to blood parasite taxon and mating system. Oikos 1998, 82:303-312.

42. Christe P, Glaizot O, Strepparava N, Devevey G, Fumagalli L: Twofold cost of reproduction: an increase in parental effort leads to higher malarial parasitaemia and to a decrease in resistance to oxidative stress. Proc $R$ Soc B 2011. doi:10.1098/rspb.2011.1546. online.

43. Bray PG, Ward SA, O'Neill PM: Quinolines and artemisinin: chemistry, biology and history. Curr Top Microbiol 2005, 295:3-38.

44. Francis SE, Sullivan DJ Jr, Goldberg DE: Hemoglobin metabolism in the malaria parasite Plasmodium falciparum. Ann Rev Microbiol 1997, 51:97-123.

45. Schwarzer E, Kühn H, Valente E, Arese P: Malaria-parasitized erythorocytes and hemozoin nonenzymatically generate large amounts of hydroxy fatty acids that inhibit monocyte functions. Blood 2003, 101:722-728. 
46. Meister A, Anderson ME: Glutathione. Ann Rev Biochem 1983, 52:711-760.

47. Chandra R, Tripathi LM, Saxena JK, Puri SK: Implication of intracellular glutathione and its related enzymes on resistance of malaria parasites to the antimalarial drug arteether. Parasitol Int 2011, 60:97-100.

48. Costantini D, Verhulst S: Does high anti-oxidant capacity indicate low oxidative stress? Funct Ecol 2009, 23:506-509.

49. Alonso-Alvarez C, Perez-Rodriguez L, Garcia JT, Viñuela J, Mateo R: Age and breeding effort as sources of individual variability in oxidative stress markers in a bird species. Physiol Biochem Zoo 2010, 83:110-118.

50. Eckman JR, Eaton JW: Dependence of plasmodial glutathione metabolism on the host cell. Nature 1979, 278:754-756.

51. Patzewitz E-M, Wong EH, Müller S: Dissecting the role of gluthathione biosynthesis in Plasmodium falciparum. Mol Microbiol 2012, 83:304-318.

52. Perrins CM: Tits and their caterpillar food supply. Ibis 1991, 133:49-54.

53. Lin S-K, Tsai S-M, Huang J-C, Lee S-C, Wu S-H, Wu S-H, Ma H, Lin J-T, Tsai L-Y: Effects of storage time and temperature on the stability of glutathione in deproteinized blood sample. J Food Drug Anal 2006, 14:141-146.

54. Becker K, Tilley L, Vennerstrom JL, Roberts D, Rogerson S, Ginsburg H: Oxidative stress in malaria parasite-infected erythrocytes: host - parasite interactions. Int J Parasitol 2004, 34:163-189.

55. Halliwell B, Gutteridge JMC: Free radicals in biology and medicine. Oxford: Clarendon Press; 2002.

56. Baker MA, Cerniglia GJ, Zaman A: Microtiter plate assay for the measurement of glutathione and glutathione disulfide in large numbers of biological samples. Anal Biochem 1990, 190:360-365.

57. Vandeputte C, Guizon I, Genestiedenis I, Vannier B, Lorenzon G: A microtiter plate assay for total glutathione and glutathione disulfide contents in cultured/isolated cells - performance study of a new miniaturized protocol. Cell Biol Toxicol 1994, 10:415-421.

58. Isaksson C, Örnborg J, Stephensen E, Andersson S: Plasma glutathione and carotenoid coloration as potential biomarkers of environmental stress in great tits. Ecohealth 2005, 2:138-146.

59. Costantini D, Dell'Omo G: Effects of T-cell-mediated immune response on avian oxidative stress. Comp Biochem Physiol A 2006, 145:137-142.

60. van de Crommenacker J, Komdeur J, Burke T, Richardson DS: Spatiotemporal variation in territory quality and oxidative status: a natural experiment in the Seychelles warbler (Acrocephalus sechellensis). J Anim Ecol 2011, 80:668-680.

61. Palacios MG, Cunnick JE, Winkler DW, Vleck CM: Immunosenescence in some but not all immune components in a free-living vertebrate, the tree swallow. Proc R Soc B 2007, 274:951-957.

62. Quinn G, Keough M: Experimental design and data analysis for biologists. Cambridge: Cambridge University Press; 2002.

63. Burnham KP, Anderson DR: Model selection and multimodel inference: $a$ practical information-theoretic approach. New York: Springer; 2002.

64. Burnham KP, Anderson DR, Huyvaert KP: AIC model selection and multimodel inference in behavioral ecology: some background, observations, and comparisons. Behav Ecol Sociobiol 2011, 65:23-35.

65. Symonds MRE, Moussalli A: A brief guide to model selection, multimodel inference and model averaging in behavioural ecology using Akaike's information criterion. Behav Ecol Sociobiol 2011, 65:13-21.

66. R Development Core Team: $R$ : A language and environment for statistical computing. Vienna, Austria: R Foundation for Statistical Computing; 2011. URL http://www.R-project.org/. ISBN 3-900051-07-0.

doi:10.1186/1472-6785-13-15

Cite this article as: Isaksson et al:: Explaining variance of avian malaria infection in the wild: the importance of host density, habitat, individual life-history and oxidative stress. BMC Ecology 2013 13:15. 